ISSN 00156043

NOTAS

\title{
La expansión del yo y la cultura de la transformación personal'
}

Joaquín P. López Novo²

Resumen: En este estudio se analiza la relevancia cultural que ha adquirido el tema de la transformación personal en las sociedades contemporáneas, analizando las orientaciones que adopta y los factores que la propician. La nueva cultura de la transformación personal unifica psicología y espiritualidad, enfatiza el poder transformador de las experiencias de trascendencia y florece en un "milieu" heterogéneo y flojamente organizado donde dominan la orientación individualizadora y la hibridación de enfoques y tradiciones. En el estudio se avanza la hipótesis interpretativa de que este desarrollo cultural es una respuesta a un proceso de cambio societal en el nivel del individuo al que denominamos la expansión del yo. El vínculo entre la expansión del yo y la cultura de la transformación personal es el malestar existencial que es inherente a la expansión del yo, para el que la cultura de la transformación personal provee una respuesta.

Palabras clave: Expansión del yo, postmaterialismo, individualización, reflexividad, malestar existencial, trascendencia, nueva espiritualidad.

Fecha de recepción: II de mayo de 201 I.

Fecha de aceptación definitiva: 6 de septiembre de $201 \mathrm{I}$.

\footnotetext{
1 Una versión preliminar de este trabajo fue presentada en un seminario celebrado en la sede de ASP en Madrid el 26 de Noviembre de 2010. Agradezco los comentarios críticos de Juan Carlos Rodríguez, Angel Sánchez Navarro, Josu Mezo, Carlos Soldevilla, José Antonio Ruiz San Román, Pablo Rodríguez Suánces, Isabela Barlinska, Santiago Pavillard, Elisa Sánchez Pérez y Víctor Pérez Díaz.

${ }^{2}$ Facultad de Ciencias Políticas y Sociología, Universidad Complutense de Madrid.
} 


\section{Expansion of the Self and the Culture of Personal Transformation}

\begin{abstract}
This study analyses the cultural significance that has been acquired by the theme of personal transformation in contemporary societies, analysing the guidelines adopted and the factors that drive it. This new culture of personal transformation unites psychology and spirituality, emphasizes the transformational power of transcendental experiences and thrives in a heterogeneous and loosely organized milieu oriented towards individualisation and the hybridisation of approaches and traditions. The study advances the hypothesis that this cultural development is a response to a process of change in society at the level of the individual, which we call self-expansion. The link between self-expansion and the culture of personal transformation is the existential malaise that is inherent in self-expansion, which finds an answer in this culture of personal transformation.
\end{abstract}

Key words: Expansion of the self, postmaterialism, individualisation, existential suffering, transcendence, new spirituality.

\section{L'expansion du moi et la culture de la transformation personnelle}

Résumé: Cette étude analyse l'importance culturelle acquise par le sujet de la transformation personnelle dans les sociétés contemporaines, en analysant les orientations adoptées et les facteurs qui la favorisent. La nouvelle culture de la transformation personnelle unifie psychologie et spiritualité, insistant sur le pouvoir transformateur des expériences de transcendance, et apparaît dans un milieu hétérogène et faiblement organisé ou prédominent les orientations individualisatrices et l'hybridation d'approches et de traditions. Cette étude avance I'hypothèse interprétative que ce développement culturel est une réponse à un processus de changement de la société au niveau de l'individu que nous appelons «l'expansion du moi». Le lien entre l'expansion du moi et la culture de la transformation personnelle est le mal-être existentiel inhérent à l'expansion du moi, auquel la culture de la transformation personnelle donne une réponse.

Mots clés: Expansion du moi, postmatérialisme, individualisation, réflexivité, mal-être existentiel, transcendance, nouvelle spiritualité.

Desde la década de los setenta del pasado siglo, estamos asistiendo al florecimientoy a la difusión de una cultura de la transformación personal centrada en la experiencia de la renovación profunda del yo, que combina terapia y espiritualidad, psicología y religión (PARTRIDGE, 2005). Esta cultura rompe con la mentalidad desencantada y materialista de la modernidad y recupera la experiencia de la trascendencia, en el contexto de una espiritualidad emancipada de la religión institucionalizada que incita a la búsqueda interior y al trabajo con uno mismo. Esta búsqueda, cuya duración temporal es indeterminada, adopta la forma de una trayectoria en la que 
el individuo, al tiempo que explora la cultura de la transformación personal, trabaja sobre sí mismo con los recursos que ésta le pone a mano (prácticas espirituales, terapias, disciplinas de trabajo corporal, medicinas alternativas, etc.). La búsqueda se orienta al crecimiento "interior" a través del trabajo consciente con uno mismo y está motivada por el afán de encontrar un "centro" en el que reafincarse y desde el que reconstruir el yo y la relación con el mundo.

Mientras que en el pasado este tipo de búsqueda solía tener lugar en el contexto de la religión institucionalizada, en el presente es cada vez más común que se oriente hacia el "milieu" de la cultura de la transformación personal, que ofrece al buscador un repertorio de prácticas y recursos para el trabajo personal en el marco de una espiritualidad emancipada de la religión institucionalizada. La posibilidad de elegir distintas opciones o caminos individualiza la búsqueda -cada quien debe encontrar su propio camino- confiriéndole la forma de un bricolage de resultados inciertos. Aunque se trata de una búsqueda espiritual, no es, sin embargo, una espiritualidad "desencarnada" que sólo mira al "más allá" y rechaza el mundo, sino una espiritualidad encarnada que se orienta hacia la vida para potenciarla, transformando las estructuras cognitivas, emocionales y energéticas del individuo, que son las tres vertientes de la transformación personal (HEELAS, 2009).

Podemos ver la cultura de la transformación personal como un idioma cultural que reinterpreta y reorienta el valor de la autorrealización del individuo, enfatizando la búsqueda personal y el trabajo transformador con uno mismo. Se ha argüido que a medida que las sociedades avanzan por la senda del desarrollo económico, acumulando riqueza y expandiendo el bienestar a todas las capas de la población, se produce un cambio de los valores que definen la ecuación del bienestar de las personas, del tal manera que los valores materialista, que enfatizan la seguridad material, van cediendo terreno a los valores postmaterialistas orientados a la cuestión de la calidad de la vida (INGLEHEART, 1997). La cultura de la transformación personal es parte de esta constelación cultural postmaterialista, porque la transformación personal es una forma de autorrealización no materialista centrada en la experiencia de la expansión interior.

Aunque el trabajo de la transformación personal tiene lugar en el contexto de la implicación del individuo con el mundo y, por tanto, no excluye la acción colectiva, difiere, sin embargo, del idioma modernista de la praxis hacedora de historia, que predica la salvación del individuo mediante la acción políitca orientada a la reconstrucción del orden social. La acción política puede promover el cambio social, pero éste no induce necesariamente la transformación personal, $y$, en ausencia de ésta, el cambio social suele ser un cambio de formas que no tocan el trasfondo de 
la existencia. La transformación personal, en cambio, es vivida como un cambio en profundidad que toca de lleno los estratos profundos de la existencia y requiere realizar un trabajo consciente con uno mismo. Por esta razón, las ideologías juegan un papel menos decisivo en la transformación personal que en el cambio social, pues lo que induce la transformación personal no es el asentimiento a una ideología, sino experiencias de expansión de la conciencia que permiten "reconocer" o "darse cuenta".

La cultura de la transformación personal también tiene afinidades con la cultura terapéutica que se desarrolló a lo largo del siglo XX de la mano de la psicología profunda y otras psicologías con un enfoque terapéutico propio (RIEFF, 1987). Pero rompe con éstas en algunos aspectos muy relevantes. Así, mientras que los enfoques terapéuticos se orientan a reconducir al individuo desde una supuesta situación de desviación o desajuste (neurosis o psicosis) a un patrón de normalidad-definido en términos de funcionamiento eficaz en el orden social establecido-, el enfoque de la transformación personal no busca reajustar al individuo al orden social existente, ni mejorar su eficacia social, sino que más bien trata de realizar su potencial personal como individuo. Por otra parte, mientras que la cultura terapéutica tiende a asumir un enfoque materialista de la psique, el enfoque de la transformación personal ve la psique como un sistema que combina elementos personales y transpersonales, que juntos dinamizan el proceso de desarrollo humano. Desde esta perspectiva, los síntomas y las aflicciones psíquicas ya no son meras manifestaciones de un funcionamiento deficiente -que habría que ser corregido mediante la intervención terapéutica apropiada-, sino que adquieren el valor de epifanías del espíritu que puede manifestarse tanto en forma de luz como de sombra (emergencias espirituales) (Groff y Groff, 1992; Assaglol, 1996)

En lo que resta de este estudio, primero hacemos un repaso de las fuentes de la cultura de la transformación personal, luego analizamos la naturaleza del "milieu" en el que está radicada y algunas de las formas sociales que adopta, y posteriormente llamamos la atención sobre los factores que han propiciado su desarrollo, poniendo un énfasis especial en un proceso de cambio societal que denominamos la "expansión del yo". 


\section{Las fuentes}

Aunque en apariencia novedosa, la cultura de la transformación personal es heredera de una tradición cultural firmemente enraizada en la historia intelectual de Occidente: el esoterismo espiritual judeo-cristiano (GOODRICK-CLARKE, 2008). Esta tradición es una corriente intelectual que a lo largo de la historia de Occidente ha articulado "una realidad alternativa", que, sin embargo, ha sido ignorada y silenciada por la historia de las ideas (TAYLOR, 1999). Un componente central de esta tradición es la experiencia de la transmutación del sujeto, entendida como un proceso de transformación de la conciencia que lo elevaría en la jerarquía del ser desde planos más densos y bajos a planos más sutiles y elevados (FAIVRE, 1994). La cultura contemporánea de la transformación personal entronca con esta preocupación de las tradiciones esotéricas por la transmutación, que también es compartida por las corrientes místicas de las grandes religiones que conciben la experiencia mística como un ascenso del alma a Dios. En el siglo XIX, bajo la influencia del avance de la ciencia y del estudio comparado de las religiones, el esoterismo adoptó una orientación secularizadora y, en la forma de la Teosofía, evolucionó hacia una ciencia de la realidad espiritual que sintetizaba una "philosophia perennis" que integraba el tronco común a todas las grandes religiones. La última ola de esta tradición cultural, y su exponente más conspicuo en nuestros días, es el movimiento cultural de la New Age (HANENGRAFF, 1998). Pero también podemos sentir un eco de esta tradición en las nuevas formas de religiosidad mística, que enfatizan la experiencia personal directa de la divinidad y el poder transformador de dicha experiencia (Cox, 1996).

El término New Age se aplica para denominar un movimiento cultural con una abundante producción literaria que apareció en la segunda mitad de la década de los setenta del pasado siglo en Inglaterra y los EE UU (FERGUSON, 1979). La New Age puede ser vista como una variante del fenómeno de la religión de la no religión: una religiosidad panteista e inmanentista que exalta la conciencia como esfera de manifestación de la divinidad y reniega de la religión institucionalizada (SPIEGLEBERG, 1943). La literatura de la New Age integra ciencia y espiritualidad, psicología y cosmología, y no faltan en ella textos pertenecientes al género de la revelación (o canalización) como el conocido "Un Curso de Milagros" (HANENGRAFF, 1998). Es particularmente llamativo el uso que hace esta corriente de la física cuántica, especialmente de las implicaciones de la naturaleza "inmaterial" de la materia (la naturaleza vibracional del "quantum") y de la naturaleza indeterminada de la realidad, lo que permite a sus sostenedores trascender las barreras que tradicionalmente han separado a la física de la metafísica y a la ciencia del misticismo (CAPRA, 1975). 
Pero la espiritualidad New Age no es la única corriente espiritual que nutre la cultura de la transformación personal, ésta también se nutre de las religiones orientales: el Hinduismo (especialmente Vedanta), las distintas variantes de Budismo (Teravada, Mahayana, Zen, etc.), el Tantrismo y el Taoismo; $y$, por supuesto, junto a éstas también están las corrientes místicas del Cristianismo y del Islam (Sufismo), y las tradiciones iniciáticas que se inspiran en ellas. Las religiones orientales, en particular, con su concepción panteista e inmanentista de la divinidad, su firme rechazo del dualismo cuerpo/mente, sus elaboradas cartografías de la psique, y el énfasis que ponen en la búsqueda de la iluminación a través de la práctica de la mediación y otras prácticas yóguicas, gozan de una influencia en la cultura de la transformación personal que rebasa el ámbito de las comunidades de sus practicantes, y han contribuido decisivamente a cincelar el vocabulario espiritual de la transformación personal, muy especialmente a través de la idea de que el desarrollo humano es un proceso de expansión de la conciencia. También han contribuido a la integración de psicología y espiritualidad, estimulando el desarrollo de psicologías transpersonales que cosmologizan la psique y dilatan el alcance de la conciencia más allá del ámbito de lo biográfico-personal (WILBER, 2000).

El énfasis en la expansión de la conciencia que caracteriza a las religiones orientales, también es compartido por los defensores del uso de enteógenos, como medios para suscitar estados expandidos de conciencia; una corriente minoritaria en la cultura de la transformación personal, pero que no podemos ignorar. Las sustancias enteogénicas pueden ser de dos tipos: sustancias sintéticas de laboratorio-también conocidos como psicodélicos-(LSD, MDMA, DMT, etc.) y preparados de plantas que producen estados alterados de conciencia (ayahuasca, peyote, hongos, San Pedro, etc.). En los años sesenta del pasado siglo, surgió en los EE UU una cultura de la psicodelia que promovía el uso de sustancias sintéticas (LSD) como recursos para el desarrollo personal y la curación psíquica (Lee, 1994). Tras la prohibición de estas sustancias en la década de los setenta, el consumo de psicodélicos se sumergió en la subcultura de las drogas, y ahí continúa todavía. También a mediados del siglo pasado, el banquero y micólogo norteamericano Robert Gordon Wasson, tras su encuentro con la curandera mejicana María Sabina, dio a conocer al publico occidental los hongos alucinógenos y sus potentes efectos visionarios, iniciando un debate sobre el papel que desempeñaron los enteógenos en la génesis de la religión (WASSON, 1972). En los años setenta, el antropólogo Carlos Castaneda, en su serie de "best sellers" sobre las enseñanzas del brujo yaqui "Don Juan", familiarizó a un extenso público occidental con los efectos visionarios del peyote (CASTANEDA, 1974). Más tarde, se hizo popular la Ayahuasca, un brebaje preparado por los curanderos del Amazonas, que también suscita estados ampliados de conciencia y visiones (MetZNer, 2005). A través de éstas y otras estas plantas, el chamanismo 
ha penetrado la cultura de la transformación personal, convirtiéndose en otro componente más de ésta, aunque minoritario (HARNER, 1990).

La cultura de la transformación personal no es sólo una constelación de creencias -un nuevo "vocabulario de motivos"-, sino también un repertorio de prácticas, pues se trata de una cultura que pone el énfasis en el poder transformador de la experiencia. Este repertorio de practicas es heteróclito y se nutre de una gran variedad de tradiciones: el yoga y las disciplinas de trabajo corporal, el repertorio de técnicas terapéuticas de las psicologías humanista y transpersonal, las prácticas de meditación de las religiones orientales (trascendental, Zen, Vipasana, etc,), las prácticas chamánicas, la canalización de energía (Reiki), la hipnosis y la videncia, el uso de la música y de la danza para generar estados de trance, la astrología y la numerología, las prácticas de sanación de la medicinas alternativas, etc. (MURPHY, 1992).

Esta amalgama de practicas revela dos atributos de la cultura de la transformación personal: su orientación ecuménica (que propicia la absorción por ósmosis de cualquier enfoque, técnica y tradición que tenga algo que aportar a la problemática de la transformación personal), y la orientación pragmática, experimental e individualizadora (que incita al individuo a experimentar con las opciones disponibles y a seguir su propio camino eligiendo lo que le vale). Esta combinación de apertura y experimentación individualizadora, favorece el bricolage espiritual por parte del individuo, así como la hibridación de las tradiciones y las escuelas, que se influyen (y se copian) recíprocamente. El bricolage es también favorecido por el énfasis en la integralidad del proceso de transformación personal, esto es: que éste tiene que tocar simultáneamente cuerpo, mente, psique y espíritu (WILEER, Patten, Leonard y MORELI: 2008)

\section{Formas sociales}

La tendencia que acabamos de mencionar es favorecida por otro hecho: que detrás de la cultura de la transformación personal no hay una organización unitaria, ni siquiera un movimiento dominante, sino una amplia variedad de corrientes que confluyen en un "milieu" que opera a la vez como catalizador de ideas y prácticas, y como mercado (Campbell, 2002). Este "milieu" comprende, entre otros, la industria editorial que difunde la literatura de la transformación personal; los medios de comunicación especializados en este "milieu" (revistas, catálogos, etc.); las librerías y las tiendas especializadas en parafernalia espiritual, que proveen 
puntos de encuentro y de información; los centros donde se imparten talleres y otras actividades de transformación personal; los gurús y los expertos que recorren el mundo impartiendo talleres y conferencias; los consultorios psicoterapéuticos que proveen servicios de terapia; las boutiques de medicinas alternativas; los centros de culto religioso con una orientación de transformación personal, etc.

El "milieu" de la cultura de la transformación personal sobrevive como parte de la economía de los servicios personales, vendiendo la transformación personal como un servicio que combina mercado y relacionalidad, racionalidad instrumental y expresividad. El taller grupal, el retiro religioso, la terapia personal y la boutiqueconsultorio son los formatos más típicos de prestación de estos servicios. Este "milieu" es simultáneamente una realidad virtual que se beneficia de las facilidades que "Internet" ofrece para la integración y la difusión de todo tipo de "milieus", por la acción de los buscadores, las redes sociales y los "links" recíprocos.

La transformación personal tiene la forma de una búsqueda jalonada de acontecimientos. El objeto de dicha búsqueda es la expansión de la conciencia y el reafincamiento existencial del individuo en la conciencia expandida; en términos existenciales, la búsqueda es una apertura hacia el interior y una profundización que dilata el horizonte existencial del individuo. En términos ideal-típicos, podemos ver esta búsqueda como un rito de paso estructurado en tres tiempos o estadios (VAn Gennep, 1960). Comienza con la decisión de "salir" de una situación existencial que el individuo suele vivir como una situación de estancamiento, bloqueo o carencia que le impele a emprender la búsqueda; a veces esta decisión toma la forma de un giro brusco a la vida de la persona, otras, en cambio, toma la forma de una secuencia de pequeños reajustes graduales. Prosigue con la fase de búsqueda y experimentación, en la que el individuo se busca a si mismo al tiempo que experimenta con el repertorio de recursos y prácticas que le ofrece la cultura de la transformación personal; ésta es la fase liminal de la búsqueda, en la que el individuo ha dejado atrás una parte de sí mismo y se afana en un trabajo de autodescubrimiento y reconstrucción del yo. Por último, si la búsqueda produce frutos, ésta acaba dando paso a una fase de reintegración, en la que el individuo se reafinca en la conciencia expandida de sí mismo y en el mundo con los frutos que le ha deparado su búsqueda. Si la búsqueda fracasa, puede cesar temporalmente, pero probablemente proseguirá en el futuro, tal vez con una nueva orientación, cuando vuelvan a darse las circunstancias propicias (TORRANCE, 1994). Por lo general, una vez que el afán de expandirse hacia adentro se activa en una persona, ese afán suele convertirse en un motivo constante que impregna y modula su vida. Pero la búsquedas varían en el modo en que "cristalizan" soluciones a los problemas de partida, y en la estabilidad de dichas soluciones. 
Los cambios de fase en el ciclo vital suelen ser circunstancias que favorecen la activación de comportamientos de búsqueda, así como las crisis vitales provocadas por pérdidas. Por otra parte, la búsqueda puede discurrir en el contexto de los marcos sociales que estructuran la vida del individuo (familia, trabajo, amigos) sin infligir cambios en ellos o alterar esos marcos (ruptura de pareja, cambio de trabajo, cambio de residencia, cambio de relaciones). En cualquier caso, la categoría del buscador no se identifica con la categoría del creyente. El buscador no busca tanto un sistema de creencias cuanto experiencias que le permitan reconstruir su yo, y su espiritualidad suele tener la forma de una "búsqueda del alma" al margen de la religión establecida, induciendo la autoidentificación como "espiritual pero no religioso" (WUTHNOW, 1998; FULLER, 2001).

La esencia de la búsqueda es el trabajo consciente con uno mismo, y dicho trabajo requiere de espacios seguros para la autoexpresión y la autoexperimentación, y dos espacios típicos para este trabajo son el taller de desarrollo personal y el retiro de meditación. Éstos pueden durar de un mínimo de un día -o un fin de semana- a una semana o más (en este caso en período vacacional), y ambos son actividades que buscan inducir experiencias de autoobservación y trascendencia, sobre todo de la "mecanicidad" de la mente y de lo que hay más allá de esa "mecanicidad". Se trata de experiencias que en el mejor de los casos pueden ser inducidas, pero nunca producidas o administradas al margen de la voluntad de los participantes; son éstos quienes las autoproducen mediante su participación activa en el juego-por así decirvaliéndose de la guía que prestan los directores o facilitadores de los trabajos.

El taller trata de fomentar la expresividad de los participantes y la autoexploración de sí mismos con métodos de "role-playing" y de comunicación franca y abierta; el hecho de que los participantes sean generalmente desconocidos unidos por un propósito común de autoconocimiento facilita soltar las inhibiciones y la labor de auto-expresión. En los talleres, la confianza se establece mediante actos recíprocos de expresión autorreveladora, que suelen tener un efecto catárquico, ya que, al tiempo que permiten al participante tomar conciencia de aspectos de sí mismo que le perturban o causan dificultades, su revelación pública en un entorno seguro le ayudan a liberarse de la culpa o la vergüenza que le producían. Por su naturaleza participativa, la necesidad de atención personalizada y las intensas dinámicas emocionales que suscitan, los talleres no pueden absorber un gran número de participantes. Este dato revela que la cultura de la transformación personal florece en grupos más bien pequeños y en encuentros que tienen la forma de acontecimientos únicos o experiencias irrepetibles, incluso cuando se trate de citas periódicas a las que el participante acude con regularidad (pues la nueva experiencia nunca es una repetición de la previa). 
El retiro de meditación, en cambio, está diseñado para propiciar una experiencia de interiorización profunda, eliminando o reduciendo al mínimo la comunicación entre los participantes, así como los estímulos externos que estos tengan que atender. Siguiendo un programa estricto en el que la actividad dominante es la meditación, el participante tiene la oportunidad de observar y soltar la actividad mental asociada a la "mecanicidad" de su ego, y verse desde una perspectiva que raramente está disponible en la vida ordinaria. En la medida en que el participante es capaz de abandonarse a la experiencia de fluir y observar los contenidos que asoman en su conciencia, entra en un estado de concentración pasiva ("samadhi") que es un estado amplificado de conciencia en el que pueden surgir iluminaciones reveladoras, y estas experiencias suelen tener un poderoso efecto liberador (despejando errores e ilusiones) y vitalizador (haciendo sentir más plenamente lo que uno es).

La transformación personal es pues una búsqueda jalonada de acontecimientos (talleres, retiros, encuentros, practicas corporales, terapias, epifanías, etc.), que proveen oportunidades para experiencias en las que la persona profundiza en el conocimiento de sí misma, comparte ese conocimiento con compañeros de viaje coparticipes en la búsqueda, y desarrolla la habilidad de explorar su mundo interior y de relacionarse consciente y responsablemente con sus contenidos. La transformación personal es, pues, una búsqueda intensamente personal y al mismo tiempo marcadamente relacional: uno no puede transformarse por sí sólo; necesita la ayuda de guías, maestros, aliados, intérpretes, compañeros y amigos que orienten, estimulen, apoyen y aporten un valioso refrendo de intersubjetividad.

La naturaleza individualizada de la búsqueda y la fuerza del mercado en la cultura de la transformación personal, tienden a inhibir la formación de comunidades sectarias "voraces", en el sentido que Lewis Coser (1978) usó este término. Por tanto, la forma organizativa dominante en la cultura de la organización personal no es la comunidad con fronteras constrictivas y criterios exigentes de pertenencia que tienden a inhibir las afiliaciones múltiples -la variante "bonding" de capital social-, si no más bien la red lábil que combina un núcleo restringido de vínculos relativamente duraderos y una periferia extensa de vínculos mas débiles y compatibles con otras afiliaciones -la variante "bridging" de capital social- (PUTNAM, 2001).

Por último, la búsqueda puede estar enmarcada en una práctica espiritual que opera como una especie de marco dominante que le confiere coherencia, o puede tener la forma de una secuencia de episodios que la persona va hilvanando siguiendo su intuición y las oportunidades que se le presentan. En cualquier caso, quienes se hayan implicados en la búsqueda de la transformación personal tienden a reconocerse recíprocamente como buscadores que comparten una meta común, más allá del camino específico que cada cual sigue para alcanzarla. Esta identidad de 
buscadores tiende puentes entre las distintas vías, prácticas y corrientes sectarias que pueblan el "milieu" de la transformación personal, y provee un metalenguaje inclusivo que expande la conversación más allá de las fronteras sectarias.

\section{Factores sociales que propician el desarrollo de la cultura de la transformación personal}

Aunque la cultura de la transformación personal no es un fenómeno novedoso, sí lo es su difusión creciente en las sociedades contemporáneas. En la sociedad actual, el idioma de la transformación personal ha dejado de ser un asunto de minorías poco visibles para extenderse a un público amplio. Para explicar este desplazamiento de la periferia al centro -o de minorías a públicos extensos- podemos aducir distintos factores. Uno de ellos -ya lo hemos mencionado antes- es el giro postmaterialista de los valores de las generaciones más jóvenes de las sociedades avanzadas, las cuales ya no parecen dispuestas a orientar sus vidas por valores de supervivencia que enfatizan la búsqueda de la seguridad y la maximización del bienestar material, sino que sienten la necesidad y el desafío de ir más allá de la experiencia de sus predecesores para explorar otras facetas de la existencia que aquellos no llegaron a percibir o no le atribuyeron la misma relevancia.

Otro factor que ha favorecido el desarrollo de la cultura de la transformación personal es la elevación del nivel cultural de la población a raíz de la universalización de la educación y del acceso masivo a la educación superior. La elevación del nivel educativo dota a los individuos de recursos cognitivos y de autocofianza para desarrollar su propia búsqueda personal con una actitud de auto-experimentación y de aprendizaje de la experiencia. Y esta búsqueda es también facilitada por la eclosión de recursos al alcance de la mano que ha traído la globalización y el desarrollo de las comunicaciones. Así, la globalización favorece la difusión "urbi et orbi" de tradiciones, ideas y prácticas que antes eran inaccesibles salvo para unos pocos especialistas, pero que hoy están al alcance de cualquiera que disponga de la necesaria curiosidad y motivación. Las facilidades para viajar a escala planetaria favorecen el desarrollo de un nuevo tipo de turismo espiritual (al Tibet, a la India, a la Amazonía, etc.) que busca un conocimiento de primera mano de tradiciones espirituales ancestrales, que ahora son directamente accesibles para el buscador curioso. Y la globalización también facilita el acceso a los enteógenos, que proveen una vía de acceso a experiencias visionarias y extáticas que desentumecen la sensibilidad espiritual de quienes han sido educados en (o han absorbido por ósmosis) la mentalidad del materialismo "cientifista". 
Otro factor que propicia la difusión del movimiento de la transformación personal es que su búsqueda posee algunos atributos intrínsecos que realzan su atractivo a los ojos de muchas personas con inquietudes existenciales. Uno de ellos es el sentimiento de profundidad o espesor existencial que aporta la búsqueda; sentimiento que libera de la seducción de la banalidad superficial y nihilista que domina buena parte de la cultura postmoderna. Otro es el sentimiento de aventura y estremecimiento que acompañan a la búsqueda; en un mundo sin fronteras, la transformación personal es una suerte de última frontera a explorar, y el hecho de que no sea una frontera geográfica sino existencial, asequible a todos los que sienten su llamada, hace que ésta sea más irresistible. Por último, la transformación personal, al reafincar a las personas en una experiencia de sí mismas que no está al albur de las circunstancias externas, puede operar como un "adaptógeno" que potencia su adaptación a un mundo en rápida transformación.

Hasta aquí hemos caracterizado la transformación personal como un proceso de expansión interior de la persona (profundización) cuya esencia es la expansión de la conciencia de si mismo y el reafincamiento en la conciencia expandida, y hemos caracterizado a grandes rasgos la cultura de la transformación personal y mencionado algunos factores que favorecen su difusión. La tesis que desarrollamos a continuación es que la cultura de la transformación personal debe ser vista en el contexto de (y como una respuesta a) el proceso expansión del yo en las sociedades contemporáneas y las dinámicas psico-existenciales que dicho proceso desata.

\section{La expansión del yo}

Por "expansión del yo" entendemos la creciente complejidad psico-existencial del yo en las sociedades contemporáneas. Podríamos decir que la expansión del yo es el crecimiento del territorio del yo, o del espacio psico-existencial sobre el que el yo interviene al realizar sus proyectos vitales. La expansión del yo es un proceso que se encuentra en el punto de intersección de biología y sociedad: de una parte, depende del nivel de energía disponible que activa deseos y aspiraciones, $y$, de otra, de las oportunidades sociales para la realización de tales deseos. Nuestra tesis es que en las sociedades contemporáneas el yo ha experimentado una expansión dramática como resultado de la concurrencia de dos cambios societales de gran alcance, uno es la prolongación de la esperanza de vida de la población y el otro es la individualización creciente de la vida social. 
En lo que concierne a la prolongación de la esperanza de vida, baste señalar dos hechos: primero, todavía a principios del siglo XX, la esperanza de vida media de las poblaciones de los países más desarrolladas era de cuarenta y pocos años; segundo, hasta finales del sigo XIX la mayoría de la población de todos los países vivía en un régimen de subnutrición crónica que a penas proveía la energía necesaria para trabajar y mantenerse (FOGEL, 2004). En otras palabras, hasta el siglo XX, para la mayoría de las personas, la vida era corta y la energía de que disponían era la justa para sobrevivir. El siglo XX, sin embargo, ha casi duplicado la esperanza de vida de las personas (en los países desarrollados ya supera o ronda los ochenta años), y, además, ha mejorado significativamente tanto la nutrición como el tiempo de ocio de que dispone la población. Hoy vivimos mucho más, disponemos de más energía de la necesaria para subsistir, llevamos vidas más saludables y disponemos de tiempo libre para explorar el mundo y explorarnos a nosotros mismos. Dado que la primera mitad del siglo XX fue una época azarosa que comprendió dos grandes guerras mundiales y una gran depresión económica entre ellas, hubo que esperar hasta la segunda mitad del siglo para ver surgir una generación que pudiese beneficiarse plenamente de un ciclo largo de estabilidad y prosperidad, y esta generación fue la de los llamados "baby boomers" nacidos en la segunda postguerra, que no por casualidad es la primera generación que dio un salto hacia delante en el proceso de expansión del yo (CLARK ROOF, 1999). No podemos entender el proceso de expansión del yo si no lo situamos en el contexto de este cambio del trasfondo energético de la vida.

Pero este primer cambio ha ocurrido en paralelo a un segundo cambio que es la individualización creciente de la vida social. Esta noción se refiere al proceso de cambio societal por el cual las sociedades han pasado de estructurarse en torno a colectividades que ejercían un fuerte control sobre sus miembros, limitando sus opciones y sus posibilidades de autoexpresión, a estructurarse en torno a individuos que cuentan con un amplio margen de elección para configurar su identidad y sus estilos de vida (ELAS, 1990). Ulrich Beck ha subrayado la faceta compulsiva de la individualización en la sociedad contemporánea, en la que los individuos se ven compelidos a construir sus vidas con la lógica del "hágaselo usted mismo" (BECK Y BECK-GERSHEIM, 2003). El resultado es la creciente intencionalización de la existencia: la vida del individuo deviene un escenario para la persecución de innumerables fines y proyectos que obligan al yo a elegir entre las opciones disponibles (o a crear sus propias opciones) y a tratarse a si mismo como un eslabón de una cadena medios/fines. Todo ello fomenta la reflexividad -el uso reflexivo del conocimiento para guiar el comportamiento- la cual es otro motor de la expansión del yo. 
Hay, pues, tres mecanismos que fomentan la expansión del yo. El primero es de naturaleza biológica o energética: la vida humana tiende naturalmente a la expansión, pues para el ser humano sobrevivir nunca es suficiente e, incluso en las circunstancias más limitadoras, existe el impulso a ir más allá de lo dado para crecer y expandirse (TORRANCE, 1994). El segundo mecanismo es el crecimiento de las opciones vitales y de las oportunidades de elección (una de las facetas de la individualización); cuanto más abundan las oportunidades vitales, mayores son las posibilidades de elegir, de cambiar los marcos de la experiencia y de modular la propia identidad, y por tanto de expandir el yo. Y el tercer mecanismo es la reflexividad (otra faceta del proceso de individualización), que intensifica la autoconciencia del individuo en cuanto sujeto decisor y fomenta la actitud de tratarse a sí mismo como un objeto moldeable. Detrás del proceso de expansión del yo siempre están estos tres mecanismos operando simultáneamente y reforzándose recíprocamente. En lo que sigue, sin embargo, pondremos el énfasis en la reflexividad como motor de expansión del yo.

La intensificación de la reflexividad marca un cambio decisivo en la relación entre individuo y sociedad, pues en la tradición del análisis sociológico lo social ha sido identificado bien con lo normativo y/o bien con lo prerreflexivo, es decir: con los valores y las normas sociales institucionalizadas en el entorno social y/o con la tradición que se absorbe por ósmosis, se da por descontada y no es objeto de cuestionamiento reflexivo. La expansión del yo supone, pues, la erosión de lo social en esas dos facetas: la normativa y la pre-reflexiva; supone, en suma, una desinstitucionalización de la vida social y de la vida personal. De ahí que uno de los correlatos de la expansión del yo sea la erosión de la tradición recibida, la pluralización de los estilos de vida y la diferenciación idiosincrásica de las trayectorias vitales.

A Simmel se debe la observación de que las instituciones de la sociedad moderna favorecen el predominio de la función intelectiva en la vida psíquica del individuo (la reflexividad), pues el intelecto es el medio por el que el yo conoce y se ajusta a las cadenas de causalidad que rigen la esfera de lo objetivo, y cuanto más extensas y complejas son dichas cadenas, mayor es el peso de la actividad intelectiva en el ajuste de la persona a la realidad objetiva (SIMmel 1990: 429). Pero el yo moderno no sólo utiliza la actividad intelectiva para relacionarse con el mundo externo, también la aplica a sí mismo para construirse y reconstruirse, tratándose a sí mismo como una esfera de objetividad y como un objeto moldeable con la aplicación de conocimiento objetivo. Esta actividad intelectiva refleja tiene dos consecuencias relevantes: una es la tendencia del yo moderno a habitar en un entorno mental de abstracciones (conceptos, cuasi-teorías y guiones lógicos) y a relacionarse con 
la realidad (externa e interna) desde la óptica de ese paquete de abstracciones; la otra es el uso de dicho paquete para auto-controlarse y auto-regularse en sus relaciones consigo mismo y con los demás. Estas dos tendencias del yo moderno acentúan el peso de la faceta mental-cognitiva en la balanza funcional del yo.

El predominio de la actividad intelectiva en el yo moderno se produce a costa del confinamiento de la actividad emocional en la esfera subjetiva y privada del individuo. En la modernidad las emociones dejan de jugar un papel significativo en la relación del individuo con la esfera de lo objetivo, y carecen de un anclaje en dicha esfera. La subjetivización y privatización de las emociones supone un pesado fardo para el yo moderno, que tiende a afrontar el mundo sinuoso de las emociones con la actitud de autocontrol intelectivo que aplica para relacionarse con la esfera de lo objetivo; de ahí que el yo moderno acuse una suerte de "incapacidad entrenada" para relacionarse con la emociones, y tienda a oscilar entre la racionalización y la represión de las mismas. Paradóiicamente, sin embargo, cuando el yo moderno experimenta choques y tensiones entre el mudo interior $y$ el mundo exterior tiende a decantarse por la interioridad como el ser más real y genuino, mientras que la exterioridad es percibida como artificial y artificiosa (TAYLOR, 1992). Este desplazamiento del eje gravitacional del yo moderno hacia la interioridad, tiene que ver con el hecho de que ésta es el territorio de las emociones, y éstas portan un acento de realidad -y una fuerza de arrastre- superior tanto a la de las racionalizaciones abstractas de la mente como a las convenciones vigentes en la realidad exterior.

Entre los factores que impulsan la individualización y, por tanto, la reflexividad del yo moderno destaca el afán de la autorrealización personal, que induce al individuo a tomar conciencia de su singularidad y a buscar dentro de sí sus valores y la dirección de los impulsos vitales que dinamizan su existencia. En la fase avanzada del proceso de modernización, no sólo crece la masa de quienes orientan sus vidas por el motivo de la autorrealización, sino que también dicho anhelo se mantiene activo a lo largo de todo el ciclo de vida de la persona. Ya no se trata de un puñado de elecciones realizadas al inicio de la vida adulta (profesión, residencia, matrimonio, etc.), que, una vez consumadas, asumen el carácter de decisiones irreversibles que marcan el resto de la vida personal. Ahora la persona vive toda su vida adulta con -por así decir- "el piloto" de la autorrealización encendido, y está dispuesta a revertir aquellas elecciones tempranas (trabajo, residencia, matrimonio, creencias, relaciones, orientación sexual, etc.) si percibe que no han sido acertadas desde la perspectiva de su autorrealización. Cuanto más peso tiene el valor de la autorrealización en la vida, más ésta adquiere el carácter de una serie de experimentos temporales sujetos a revisión, y más las personas están 
dispuestas a darse oportunidades adicionales de experimentación; cada nueva etapa del ciclo vital es una oportunidad para revisar el modo de autorrealización de la persona y para ensayar nuevas formas. La dilatación del alcance temporal del anhelo de autorrealización induce un cuestionamiento incesante de la interioridad para descubrir el yo auténtico que yace oculto bajo los errores del pasado, los compromisos caducos, las represiones y las falsas ilusiones; y esta interrogación incesante de la interioridad es otro motor de expansión del yo.

Pero el yo no sólo vive para sí mismo, sino que es social y necesita a los otros. El yo siempre está situado en una red social de interdependencias que le obligan a responder a las demandas de los otros, y a vigilar los efectos que producen sus acciones en aquellos de los que depende (Elias, 1987). En un ensayo que tuvo un gran impacto a mediados del siglo pasado, David Riesman (1950) distinguió tres tipos ideales de carácter social: el tradicionalista, el autodirigido (o dirigido por sus impulsos interiores), y el dirigido por los otros ("other regarding"); detrás de esta tipología estaba la crítica de Riesman a la nueva sociedad de masas, que supuestamente producía tipos humanos "other regarding" carentes de iniciativa y fibra moral; pero lo cierto es que el yo moderno, por muy dotado que esté de iniciativa e independencia de juicio, no puede ser indiferente al impacto de su conducta en los otros, y por ello está condenado a ser "other regarding". Es más, como bien señaló Erving Goffman, la vida social, por su naturaleza relacional e interactiva, conlleva un trabajo incesante de presentación de la persona, de puesta en escena; un trabajo que se hace con dosis variables de reflexividad, dependiendo del contexto y de lo que está en juego en la situación de interacción (GOFFMAN, 1959). La modernidad intensifica la reflexividad del trabajo de presentación de la persona, porque en el mundo moderno la reputación personal se asienta sobre criterios performativos en vez de posicionales, y la imagen que la persona proyecta ante los otros suele tener un peso decisivo en la formación de su reputación. Por esta razón, las personas cuidan esta imagen y se esfuerzan en modelarla eligiendo cuidadosamente sus modos de presentación en público. En suma, la intensificación de la reflexividad inherente a la presentación pública del yo es otro factor de expansión del yo.

Pero la reflexividad no se limita al aspecto teatral o de puesta en escena de la acción, sino que toca de lleno los aspectos sustantivos de la acción. La modernidad constituye a los individuos "qua" actores, esto es: sujetos dotados de capacidades de agencia que emplean para perseguir propósitos racionales, valiéndose de conocimiento (formal y tácito) de la relación medios/fines (MEYER Y JEPPERSON, 2000). La acción racional orientada por un propósito, que es la marca distintiva del individuo "qua" actor, es otra fuente de reflexividad que potencia la expansión del yo, y ello 
no sólo porque obliga al individuo a operar como una fuerza causal productora de efectos intencionados, sino también porque induce al individuo a trabajar reflexivamente sobre si mismo, tanto en el plano de los motivos de la acción, como en el de las capacidades necesarias para actuar con eficacia. Los actores de la sociedad moderna están obligados a auto-capacitarse sin fin, como pone de manifiesto el desarrollo de la cultura de los Recursos Humanos; y esa autocapacitación tiene lugar mediante procesos de formación que no sólo aportan nuevos recursos y habilidades, sino que también cincelan la autoimagen de la persona.

Incluso la acción afectiva, que en el paradigma Weberiano no se orienta por criterios racionales, en la modernidad, sin embargo, se carga de reflexividad y los afectos y las emociones son objeto de observación e interrogación (EHRENBERG, 2008).

Por último, otro factor que contribuye a la expansión del yo por la vía de la intensificación de la reflexividad es el dinamismo de la vida social. El cambio sociocultural socava la tradición y muta los acuerdos pre-reflexivos que sostenían los mores heredados, abriendo la puerta a nuevos modos de acción y relación que traen consigo nuevas actitudes e identidades, y también nuevas demandas que saturan la capacidad de ajuste del yo (GERGEN, 1998) Al mutar los contextos de la vida social, el cambio social obliga al individuo a reposicionarse en nuevas formas sociales, descartando viejos guiones periclitados e internalizando nuevos guiones que redefinen el marco de lo que es correcto y apropiado. Este "aggiornamento" es reflexivo y torna al individuo más consciente de la construcción social de la realidad, esto es: de que ésta se basa en acuerdos compartidos frágiles y mutables. El dinamismo social también puede erosionar la seguridad básica (óntica) que sostiene al yo, precipitando espirales de reflexividad para restaurarla (GIDDENS, 1991). Podemos afirmar que cuanto más el dinamismo social socava y transforma los límites y las fronteras de la realidad social en que se desenvuelve el individuo, más éste siente la necesidad de afincarse en una experiencia interior que le aporte una seguridad existencial al abrigo de las mutaciones que experimentan las fronteras exteriores. En definitiva, el cambio social intensifica la conciencia de la separación entre el mundo interior y el mundo exterior (entre esencia y máscara) e induce al individuo a buscar en sí mismo puntos de apoyo que le mantengan al abrigo de las mutaciones externas; $y$ estos dos efectos amplifican la expansión del yo.

Llegados a este punto hemos de preguntarnos: ¿qué consecuencias tiene la expansión del yo? , y ¿̇por qué dicha expansión es relevante para el desarrollo de la cultura de la transformación personal? La tesis que aquí proponemos es que cuanto más se expande el yo más éste tiene que auto-construirse mediante su propia agencia reflexiva, y uno de los motivos de fondo que orientan este trabajo de auto-cons- 
trucción es el malestar existencial que el yo expandido tiende a experimentar en razón de su complejidad y su posible (y probable) incoherencia.

\section{Expansión del yo y malestar existencial}

La característica más definitoria del yo expandido es pues su auto-constructivismo reflexivo. El yo expandido se ve impelido a hacerse a si mismo mediante elecciones, de tal manera que su identidad, estilo expresivo, opciones vitales y orientaciones existenciales son el producto de elecciones - a veces tácitas otras explícitas- siempre reversibles. El constructivismo del yo expandido tiende a favorecer una actitud de auto-control mental en la que el yo se trata a si mismo como un objeto moldeable, aplicando a sus contenidos la lógica de la racionalidad instrumental. El resultado es una autoimagen racionalizada que choca con la naturaleza viscosa y recalcitrante de las emociones y los estados de ánimo, y con la reactividad mecánica de la mente (TART, 1986). El choque entre la racionalidad constructivista "egótica" y las dinámicas emocionales reactivas que se sustraen a la voluntad del yo, es la matriz de experiencias de "yo divido" que causan sufrimiento a la persona e inducen comportamientos de búsqueda para aliviar el sufrimiento. Un yo expandido es inevitablemente un yo crónicamente inquieto, embarcado en el proyecto modernista de autoconstrucción racional que más pronto o más tarde topa con límites que inducen el auto-cuestionamiento y activan comportamientos de búsqueda para su auto-reconstrucción.

El proyecto modernista de autoconstrucción reflexiva del yo opera con una lógica modular, que induce la autorregulación de los individuos para que éstos operen como módulos adaptables en las familias, las organizaciones y otras redes cooperativas (GELLNER, 1996). Pero esta lógica modular deja sin responder la cuestión existencial del sentido de la existencia. En nuestra opinión, la inestabilidad inherente al yo expandido hace que más pronto o más tarde el problema existencial del sentido adquiera relevancia para el individuo, volviéndose más central en sus preocupaciones e induciéndolo a emprender búsquedas. El yo expandido es proclive a los sentimientos de pérdida de sentido (GIDDENS, 1991) y los cambios de fase en el ciclo vital suelen operar como resortes activadores que sacan a la luz el problema del sentido y lo ponen en el centro de las preocupaciones del individuo.

Un yo expandido es también un yo que tiene una conciencia aguda de su propia complejidad, tanto en lo que concierne a la variedad de estados emocionales que atraviesa como a la multiplicidad de entornos ("mundos parciales") que tiene que 
conciliar. Un yo expandido es, pues, un yo que se siente en alguna medida como un extraño para si mismo y ansía conocerse e integrarse; se ve a si mismo como una "terra incognita" que ha de ser explorada, y el motivo que incita esta exploración no es sólo -ni fundamentalmente- la curiosidad, sino el malestar que acompaña a la experiencia de la ausencia de un centro que integre y ordene armónicamente los distintos elementos del yo, junto al presentimiento de que ese centro existe y que es responsabilidad del yo buscarlo y afincarse en él.

El malestar existencial del yo expandido trasciende la distinción entre lo normal y lo patológico. En su estudio clásico sobre las Variedades de Experiencia Religiosa, William James afirmó que los seres humanos pueden ser clasificados en dos grandes tipos: los nacidos una sola vez y aquellos que han de nacer dos veces. El yo expandido pertenece a la segunda categoría; es un yo que se siente incompleto y ansía completarse, y para ello se ve impelido a hacer una labor de búsqueda y transformación interior, pues lo medios que le ofrece la cultura para autorrealizarse (trabajo y familia, roles y relaciones) no le bastan. Podríamos decir que un yo expandido es un yo que siente que una parte de su tarea vital es evolucionar, crecer, y hacerlo de manera voluntarista, esto es: mediante un trabajo de auto-transformación.

\section{Expansión del yo y sufrimiento existencial}

Se ha argüido que el desarrollo económico y, sobre todo, el desarrollo del estado de bienestar en la segunda mitad del siglo XX, ha incrementado de manera tan significativa el nivel de seguridad existencial de las poblaciones de las sociedades más avanzadas, que este factor (medido por indicadores como el Índice de Desarrollo Humano de las Naciones Unidas) sería la principal variable que explicaría el declive de la religión en dichas sociedades (y su persistencia en las sociedades más atrasadas) (NORRIS E INGLEHART, 2004; HUBBER Y KRECHM, 2009). Pero aún admitiendo que el desarrollo del estado de bienestar ha mejorado significativamente algunas facetas de la seguridad existencial (por ejemplo, protegiendo al individuo frente a las contingencias del desempleo, la enfermedad y la vejez), es dudoso que haya eliminado el malestar existencial.

Nuestra argumento resalta que el malestar existencial es inherente a la expansión del yo y que el aumento de la seguridad existencial en ciertas dimensiones de la vida no supone la erradicación del malestar existencial, sino su metamorfosis. La expansión del yo incrementa la susceptibilidad del individuo al malestar existencial 
porque el crecimiento de la complejidad del yo no se acompaña automáticamente de una integración satisfactoria de dicha complejidad en el plano psico-existencial, sino que ha de ser el individuo quien la realice. Puesto que la expansión del yo tiende a inducir una mayor autoconciencia en el individuo, ésta también es un espejo que refleja el malestar existencial que acarrea; malestar que brota de múltiples fuentes: la falta de una respuesta al problema del sentido; el sufrimiento psicológico que genera la mente reactiva; el estrés que producen las pérdidas y la incertidumbre; la precariedad (impermanencia) de los afectos y las relaciones; la conciencia de carencia en medio de la abundancia, muy especialmente de la carencia de un centro interior que integre y armonice la experiencia; y también sentimientos difusos de carencia: de que algo falta en la vida, aunque no se acabe de ver o entender qué es (Lor, 2000). Peter Berger utilizóla expresión "homelessness" (pérdida de hogar) para caracterizar esta conciencia de carencia, caracterizada por el sentimiento de la pérdida de un hogar metafísico (cósmico) y el anhelo de recuperarlo (BERGER, BERGER y KeLLNER, 1974). Aquí, sin embargo, preferimos utilizar el término descentramiento para referirnos al malestar existencial que arrostra la expansión del yo: el sentimiento de carencia de un centro unificador y el anhelo de re-centrarse.

En este estudio resaltamos esta faceta del malestar existencial como correlato del proceso de expansión del yo, porque la sociología, por lo general, ha sido ciega ante el dolor existencial, procediendo sobre el supuesto tácito de que el único sufrimiento merecedor de atención es el que se origina en causas sociales (desigualdad, opresión social, explotación, exclusión, etc.) (BOURDIEU, 1999). Pero aunque el dolor existencial no es social en el sentido estricto del término, sí posee relevancia sociológica porque es un poderoso resorte motivador. Es más, podemos afirmar que una de las funciones básicas del orden social en todo tiempo y lugar es la defensa del individuo frente a las embestidas del dolor existencial, dotándolo de una identidad social y de obligaciones sociales que ocupan su tiempo y su energía, y reforzando con la fuerza de lo social los mecanismos de defensa psicológicos que contienen la ansiedad existencial. La creciente individualización de la vida social, sin embargo, merma la capacidad del orden social de actuar como barrera de contención de la ansiedad y el malestar existencial, al tiempo que la mayor autoconciencia del individuo los amplifican. He aquí, pues, el factor estructural que provee un contexto de plausibilidad para la difusión del idioma cultural de la transformación personal en las sociedades contemporáneas.

Lo que distingue a la cultura de la transformación personal es su enfoque del malestar existencial como la ocasión para despertar a una conciencia superior y como un síntoma positivo que indica que dicho despertar ya se ha iniciado y está 
en marcha. La cultura de la transformación personal ve la expansión del yo como parte y parcela de un proceso de evolución de la conciencia y llama al individuo a asumir un papel activo en ese proceso, esforzándose en expandir su propia autoconciencia (HANENGRAAFF, 1998).

La cultura de la transformación personal enfatiza la responsabilidad del individuo por su propia transformación personal a través de un proceso de búsqueda, experimentación y aprendizaje de la experiencia. En esta búsqueda, las experiencias de trascendencia (las vivencias de ausencia de límites) juegan un papel decisivo como mecanismos de reafincamiento en un estrato existencial al abrigo de los procesos psicológicos generadores de sufrimiento. A la vista de la importancia que revisten estas experiencias añadiremos algunas consideraciones sobre su estructura.

\section{Autotrascendencia y reafincamiento}

La reflexividad del yo incluye dos capacidades críticas para el proceso de transformación personal: la capacidad de trascendencia y la capacidad de re-afincarse en trascendencias. En lo que concierne a la primera, ésta es la capacidad del yo de ir más allá de lo inmediatamente dado en la experiencia, bien interpretando los contenidos de ésta como símbolos que apuntan a una realidad subyacente a la que el yo atribuye significado, o bien desplazando el foco de atención de los contenidos específicos de la experiencia al continente en el que dichos contenidos están -por así decir-suspendidos (esto es: de la figura al fondo). La primera es una habilidad hermenéutica que articula conexiones entre distintos planos de realidad (visible e invisible), la segunda es una habilidad contemplativa que disuelve los límites del yo en el trasfondo de la experiencia. Operando de manera separada o combinada, estas dos habilidades cimientan las experiencias de autotrascedencia del yo. Estas experiencias varían en su contenido y en su alcance o inclusividad, ya que el yo siempre está afincado en una constelación de trascendencias.

El sociólogo de la religión Thomas Luckmann (1990) ha clasificado las trascendencias en tres grandes categorías, atendiendo al criterio de su inclusividad. Las menos inclusivas son las pequeñas trascendencias de la vida personal (la autoimagen personal, el alma, los mundos compartidos de las relaciones íntimas); conviene recordar que la identidad personal es una forma de auto-trascendencia, pues es una narrativa (esto es: una interpretación) que impone orden y sentido a los contenidos primarios de la experiencia biográfica del individuo, que habrían podido ser ordenados (interpretados) de otra manera y que, de hecho, el individuo puede 
reinterpretar en el transcurso del tiempo, a medida que la acumulación de experiencia cambia la perspectiva desde la que contempla su trayectoria vital. En el siguiente nivel de inclusividad están las transcendencias medianas que configuran el imaginario social que da sentido a la vida en colectividad (familia, comunidades, clases, etnias, nación, etc.). Por último, están las grandes trascendencias cósmicas que constituyen el imaginario religioso, y que pueden ser tematizadas bien en términos teistas (como una alteridad trascendente hacia la que podemos orientarnos dialógicamente), bien en términos panteistas e inmanentistas (como una fuerza cósmica creadora omnipresente en su creación y, por tanto, en uno mismo en cuanto parte de dicha creación). Luckmann confeccionó esta clasificación con el propósito de argumentar que la cultura desencantada de la modernidad no suprime la trascendencia, sino que la encoge; problematiza las grandes trascendencias cósmico-religiosas (las canopias sagradas), pero refuerza las pequeñas trascendencias de la vida personal y las medianas del imaginario social. Pero las trascendencias seculares de la modernidad siguen siendo trascendencias, fundadas en la capacidad de autotrascendencia del yo.

La cultura de la transformación personal representa un retorno de la gran trascendencia, incluyendo un renacimiento de la cosmología. Pero este retorno no es un regreso a formas de religiosidad convencional, sino que conlleva un énfasis novedoso en la experiencia directa del trasfondo sin límites que subyace a nuestra experiencia de un universo mental y social estructurado por límites. Afincando su conciencia en ese trasfondo sin limites -la "gran mente" y el "gran corazón" de la tradición budista (GeMPo Merzel, 2007)-, el individuo se asienta en un estrato existencial que abarca y al mismo tiempo trasciende las dinámicas psicológicas y sociales que son el producto de una realidad (mental, corporal y social) estructurada por límites. Al afincarse en la conciencia de ausencia de límites, el individuo se conecta con la corriente profunda de la existencia y cesa de vivir los limites que estructuran su vida como una prisión opresiva. Esta transformación empodera al individuo y potencia su adaptabilidad a un entorno en continua mutación. La forma privilegiada para acceder a esa experiencia de no-límites es la experiencia de la conciencia del "ahora" (Tolle, 2000).

El énfasis en la vivencia de la ausencia de límites en el trasfondo de la experiencia ordinaria desplaza el "locus" de la trascendencia de un más allá cósmico, que es un asunto de creencia, a un más allá existencial que ya no es un asunto de mera creencia sino de experiencia. En definitiva, en la cultura de la transformación personal, la puerta de acceso a lo trascendente está en el yo, no fuera de él. En este contexto, la creencia juega un papel en la elaboración ex post de la experiencia, pero no es un requisito de la misma; la creencia ya no es un requisito de la fe. 
El énfasis en la expansión de la conciencia acarrea una revalorización del inconsciente, que deja de ser visto - a la manera freudiana- como un contenedor de instintos antisociales y de detritus psíquico, que ha de ser reprimido por el bien del individuo y la sociedad, y pasa a ser visto como el hogar de fuerzas vitales creativas que dinamizan la existencia y que aguardan su acceso a la conciencia (Fuller, 1986). Se trata de un inconsciente que es mucho más extenso que el inconsciente personal freudiano, e incluso que el inconsciente colectivo jungiano. La cultura de la transformación personal ve las corrientes vitales del inconsciente que resuenan en la conciencia como fuerzas vitalizadoras que tienen el poder de renovar, aportando inspiración, flexibilidad y fortaleza; son, por tanto, adaptógenos que potencian la capacidad de adaptación del yo, ayudándolo a desenvolverse en contextos caracterizados por la aceleración del cambio.

\section{Límites y contradicciones de la cultura de la transformación personal}

En este estudio hemos llamado la atención sobre un cambio cultural que está teniendo lugar en las sociedades avanzadas, pero que tiene visos de ser un cambio de alcance global que se extenderá y profundizará en el futuro. Se trata del desarrollo de una cultura de la transformación personal que aporta medios para reafincar al individuo en experiencias de trascendencia que lo renuevan y vigorizan, potenciando su autonomía y su capacidad de agencia responsable. Aunque no creemos que sea posible explicar este cambio apelando única y exclusivamente a factores de naturaleza sociológica, algunos factores de este tipo lo están propiciando y continuarán haciéndolo en el futuro. Entre ellos hemos subrayado el proceso de expansión del yo, que es el producto de un cambio societal de larga duración y probablemente irreversible. La expansión del yo impele al individuo a autoconstruirse con los materiales que encuentra a su alcance con vistas a su autorrealización; pero el sufrimiento existencial y emocional que es inherente a la autoconstrucción del yo es una fuente de inestabilidad que induce procesos de búsqueda. La cultura de la transformación personal ofrece una respuesta a los anhelos de búsqueda del yo expandido; esta respuesta enfatiza la expansión interior como un medio para el recentramiento del yo, a través de experiencias que permiten al yo autotrascender las fronteras mentales que lo aprisionan. La cultura de la transformación personal provee al yo expandido medios y recursos para realizar la experiencia del "segundo nacimiento", que lo libera de la esclavitud de la mente reactiva. 
La cultura de la transformación personal es una cultura emancipadora pues trata de liberar al individuo de las cadenas que le mantienen atado a patrones inconscientes, así como de las ilusiones y las compulsiones de su ego. Promoviendo el desarrollo de la conciencia, esta cultura contribuye a forjar individuos más libres y poseídos de sí mismos, y por tanto más responsables. Como el motivo dominante de quienes han renacido a una identidad más profunda suele ser servir a los demás en el mejor modo posible, la cultura de la transformación personal tiende a favorecer la difusión de orientaciones altruistas y de una ética del servicio.

Pero no queremos concluir este estudio sin señalar también algunos puntos débiles de este desarrollo cultural. En realidad, estos puntos débiles no son sino la otra cara de sus puntos fuertes (o su "sombra", entendiendo esta expresión en el sentido que Jung le atribuye). Así, la cultura de la transformación personal es una cultura individualizadora con un fuerte componente anti-institucional que pone el énfasis en la experiencia directa del individuo y en la libertad de éste para experimentar y seguir su propio camino. Las comunidades que se forman en su seno son comunidades circunstanciales de compañeros de viaje, que hacen un trecho juntos antes de volver a separarse para proseguir cada uno el viaje a su manera y ritmo; son como bandadas de pájaros que continuamente se deshacen para rehacerse con otras formas y composiciones. Estas comunidades intermitentes tiene poco que ver con la organización sectaria que opera como una organización voraz y exige máxima lealtad y entrega a sus miembros. Pero esta orientación individualizadora también tiende a favorecer la superficialidad y el desarrollo de una actitud de consumismo espiritual que Chogyam Trungpa (2008) denominó materialismo espiritual. Actitud ésta que también es favorecida por la orientación hacia el mercado del "milieu" de la transformación personal, que propicia la percepción de ésta como una mercancía consumible a precios de mercado.

Otro aspecto valioso de la cultura de la transformación personal es su orientación antidogmática, fruto del énfasis que pone en la experiencia directa del individuo como criterio de veracidad y relevancia, lo que se traduce en el tratamiento flexible de las creencias y en la apertura a la experimentación de nuevas ideas y prácticas procedentes de tradiciones diversas. La otra cara de la moneda de esta tendencia a la hibridación es la cristalización de pastiches heteróclitos y poco coherentes, vertebrados por el espíritu del oportunismo.

La cultura de la transformación personal recupera la experiencia de la trascendencia y hace de dicha experiencia el pivote de la transformación personal, pero en muchas ocasiones se trata de experiencias de trascendencia rudas y precarias 
("misticismo crudo") porque la experiencia de no límites tiene sus riesgos y no necesariamente conduce a la integración en un nivel de conciencia superior; sobre todo, se trata de experiencias de trascendencia que no inducen la afirmación de valores trascendentes, y por tanto carecen del poder de transformar el orden social mediante la afirmación de nuevos valores. En este sentido, la cultura de la transformación personal es tributaria de la cultura secularista de la descreencia, como pone de manifiesto la alergia a la religión organizada que suelen exhibir los representantes de esta cultura.

La cultura de la transformación personal es una espiritualidad del yo, que incita al individuo a buscar en sí mismo recursos latentes y a llevar a cabo una renovación profunda con dichos recursos; esa renovación, al hacer al individuo más libre y responsable, no sólo le beneficia a él, sino también a la colectividad, porque la transformación personal es afín a la ética del servicio. Pero la cultura de la transformación personal carece de un plan para la reconstrucción del orden social; no hay en ella un esbozo de ingeniería social; tal vez porque el empoderamiento del individuo que busca esta cultura hace menos necesaria la ingeniería social, que siempre contempla al individuo como una partícula de la masa, y no como un agente creativo y responsable. Si acaso, la cultura de la transformación personal propicia la experimentación en pequeña escala, así como el espíritu empresarial. Sea como fuere, hay quienes piensan, y tal vez no les falte razón, que el concernimiento con la transformación del individuo puede favorecer actitudes "narcisistas" de auto-contemplación del propio ombligo no del todo saludables.

Tal vez la mayor contribución de la cultura de la transformación personal es su función de laboratorio de invención, reinvención y puesta a prueba de ideas y prácticas para actualizar el potencial del individuo. De ese proceso de experimentación brotan innovaciones (ideas y prácticas) que acaban penetrando y difundiéndose en la sociedad, y cambiando valores, hábitos y estilos.

\section{Referencias bibliográficas}

BeCK, U. y BeCK-GERSHEIM, E. (2003) La Individualización. El individualismo institucionalizado y sus consecuencias sociales y políticas. Barcelona: Piados.

Berger, Peter L., Berger, Brigitte y Kellner, H. (1974) The Homeless Mind. Modernization and consciousness. New York: Vintage. 
Bourdieu, P. (1999) La Miseria del Mundo. Madrid: Akal.

CAMPBELL, C. (2002) "The cult, the cultic milieu and secularization", en Jeffrey KaPLAN y Helene Lööw (eds) The Cultic Miilieu. Oppositional Subcultures in an Age of Globalization. Walnut Creek (Ca): Altamira Press, pp: 12-26.

CAPRA, F. (1975) The Tao of Physics: An Exploration of the Parallels Between Modern Physics and Eastern Mysticism. Boulder (Co.): Shambala.

Castaneda, C. (1974) Las Enseñanzas de Don Juan: Una forma Yaqui de conocimiento. México (D. F.): Fondo de Cultura Económica.

Clark RoOF, W. (1999) The Spiritual Marketplace. Babyboomers and the Remaking of American Religion. Princeton (NJ): Princeton University Press.

Coser, L. A. (1978) Las Instituciones Voraces. México. Fondo de Cultura Económica.

Cox, H. G. (1999) Fire from Heaven: The Rise of Pentecostal Spirituality and the Reshaping of Religión in the 21 st Centrury. London: Cassell.

Ehrenberg, A. (2008) La Fatigue d'Etre Soi. Depresión et Societé. París: Odile Jacob.

ElAs, N. (1990) La Sociedad de los Individuos. Barcelona: Península.

Falvre, A. (1994) Access to Western Esotericism. New Yor: New York University Press.

Ferguson, M. (1979) The Aquarian Conspiracy. Personal and Social Transformartion in Our Time. New York: J. P. Tarcher, Inc.

Fuller, R. C. (1986) Americans and the Unconscious. New York, Oxford: Oxford University Press.

- (2001) Spiritual but not Religious. Understanding Unchurched America. Oxford, New York: Oxford University Press.

GellNer, E. (1996) La Sociedad Civil y sus Rivales. Barcelona: Paidós. 
Gempo Merzel, D. (2007) Big Mind, Big Heart. Finding Your Way. Mc Lean (Virginia): Big Mind Publishing.

Gergen, K. (1998) El Yo Saturado. Dilemas de la Identidad en el Mundo Contemporáneo. Barcelona: Piados.

GIDDENS, A. (1991) Modernity and Self-Identity. The Self and Society in Late Modernity. London: Blakwell.

Goodrick-Clarke, N. (2008) The Western Esoteric Traditions. A historical introduction. Oxford: Oxford University Press.

Goffman, E. (1959) The Presentation of Self in Everyday Life. Garden City (NY): Anchor Books.

Groff, C. y Groff, S. (1992) The Stormy Search for the Self: A guide to personal growth through transformational crisis. New YorK: Jeremy P. Tarcher.

Hanegraaft, W. J. (1998) New Age Religión and Western Culture. Esotericism in the Mirrior of Secular Thought. New York: New York University Press.

Heelas, P. (2009) "Spiritualities of Life", en: Peter B. Clark (ed.), The Sociology of Religion. Oxford (UK): Oxford University Press, pp. 758-783.

Harner, M. (1990) The Way of the Shaman. New York: Harper.

HUBER, S. y KRECH, V. (2009) "The Religious field betwen globalization and regionalization", en Bertelsmann StifTUng (ed.), What the World Believes: Analysis and comentary of the religious monitor 2008. Verlag Bertelsmann Stiftung, pp.: 53-95.

INGelHEART, R. (1997) Modernization and Postmodernization. Cultural, Economic and Political Change in 43 Societies. Princeton (NJ): Princeton University Press.

KRIPAL, J. J. (2007) Esalen. America and the Religión of No Religión. Chicago: The University of Chicago Press.

LaCHMAN, G. (2003) A Secret History of Consciousness. Great Barrington (Mass): Lindisfarne Books. 
LEE, M. A. (1994) Acid Dreams: The Complete Social History of LSD. New York: Groove Press.

LoY, D. (2000) Lack and Transcendence. The problem of death and life in psycotherapy, existentialism, and Buddism. New York: Humanities Books.

LUCKMANN, T. (1990) "Shrinking Transcendence. Expanding Religión?", Sociological Análisis, vol 50 (2): 127-138.

Metzner, R. (2005) Sacred Vine of the Spirit: Ayahuasca. South Paris (Maine): Park Street Press.

MeYer, J. W. y R. L. JePPERSON (2000) "The "actors" of modern society: the cultural construction of social agency", Sociological Theory, vol 18 (1), pp. 100-20.

MURPHY, M. (1992) The Future of the Body. Explorations into the future evolution of human nature. New York: G. P. Putnam's Sons.

NORRIS, P.y INGLEHART, R. (2004) Sacred and Secular. Religión and politics worldwide. New York: Cambridge University Press.

Partridge, C. (2005) Re-enchantment of the West: Alternative spiritualities, sacralization, popular culture and occulture, Vols 1 and 2. London: T \& T Clark.

Putnam, R. D. (2001) Bowling Alone: The Collapse and cevival of American Community. New York: Simon and Schuster.

RIEFF, P. (1987) The Triumph of the Therapeutic. Uses of faith after Freud. Chicago: Chicago University Press.

RIESMAN, D. (1950) The Lonely Crowd: A study in the changing American character. New York: The Free Press.

Simmel, G. (1990) The Philosophy of Money (edited by David Frisby) London and New YorK: Routledge.

SpieGelBerg, F. (1943) The Religion of No Religion. Stanford (Ca.): James Ladd Delkin.

TART, C. T. (1986) Waking Up. Overcoming the obstacles to human potential. Boston. Shambala. 
TAYLOR, C. (1992) Sources of the Self. The Making of the Modern Identity. Cambridge (Mass.): Harvard University Press.

TAYLOR, E. (1999), Shadow Culture. Psychology and Spirituality in America. Washintong D. C.: Couterpoint.

ToRRANCE, R. M. (1994) The Spiritual Quest. Transcendence in Myth, Religión, and Science. Oxford: Oxford University Press.

Van Gennep, A. (1960) The Rites of Passage. London: Routledge.

WASSON, R. G. (1972) Soma: Divine Mushroom of Inmortality. New York: Harcourt.

WILBER, K. (2000) Integral Psychology: Conciousness, Spirit, Psychology, Therapy. Boston: Shambala.

Wilber, K., Patten, T., Leonar, A. y Morelly, M. (2008) Integral Practice: A 21 stCentury Blueprint for Physical Health, Emotional Balance, Mental Clarity, and Spiritual Balance. Boston and London: Integral Books.

WUTHNOW, R. (1998) After Haeven. Spirituality in America Since the 1950's. Berkeley: Univeristy of California Press. 